\title{
Sensitivity Analysis on Service-Driven Network Planning
}

\author{
Paolo Di Francesco Member, IEEE, Jacek Kibiłda Member, IEEE, Francesco Malandrino, Member, IEEE, \\ Nicholas Kaminski, Member, IEEE, Luiz A. DaSilva, Fellow, IEEE
}

\begin{abstract}
Service providers are expected to play an increasingly central role in the mobile market and their relationship with the traditional mobile network operators (MNOs) is starting to change. The dilemma faced by over-the-top service-providers (OTT) is now whether to enter into a service level agreement with the MNOs (in the same spirit of mobile virtual network operator agreements) or to invest in deploying their own network infrastructure to serve their demand. The purpose of this paper is to study the factors shaping the agreements between OTTs and MNOs and how these factors impact network planning decisions. To this end, we build a synthetic model of cellular network deployment that explores how traditional mobile operators and OTTs compete in deploying new infrastructure. Using our model in conjunction with real-world data, we find that service-driven networks are heavily influenced by regulatory decisions, and that cost structures and demand characteristics play non-marginal roles in the definition of service-driven networks.
\end{abstract}

Index Terms-Network Planning, SLA, Sensitivity Analysis, Optimization.

\section{INTRODUCTION}

$\mathrm{T}$ HE mobile market is rapidly changing and becoming more complex. Nowadays mobile network operators' (MNOs) ability to generate revenue relies, firstly, on their subscribers and, secondly, on wholesale agreements with mobile virtual network operators (MVNOs) in a secondtier market [1]. Unfortunately this revenue model does not seem sustainable as the growing demand for capacity and data-rates forces MNOs to heavily invest in costly network infrastructure expansions and upgrades, impacting the profitability of running a mobile network. As a result, MNOs are showing interest in different business models [2].

Meanwhile, many over-the-top service providers (OTTs) have based their success on the users' perception of limitless traffic [3] and Internet's ubiquitous access. Mobile capacity shortages, and subsequent service degradation, would affect OTTs' ability to generate profit. In particular, the OTTs offering bandwidth-intensive services such as HD video streaming on-demand or online gaming, which require strict quality of service (QoS), are the most exposed. Essentially, these OTTs are presented with two (non-exclusive) strategies: (i) to acquire capacity on-demand from MNOs, and (ii) to deploy their own infrastructure. Indeed we are already starting to witness similar scenarios. For example, Google's Project Fi [4] offers to its subscribers both Wi-Fi, as part of Google's effort to deploy its own infrastructure, and LTE connection, as part of Google's MVNO agreement with traditional MNOs (i.e., Sprint and T-Mobile in the US). Other examples also exist and include the FreeBasics initiative by Facebook [5] and the Twitter deals [6].

A third strategy exists and aims at acting on the traffic demand. In its simplest form, certain types of traffic are charged at higher rates or downright forbidden. We leave

- All authors are with CONNECT, Trinity College Dublin, Ireland.

This publication has emanated from research supported in part by research grants from Science Foundation Ireland (SFI) under Grant Numbers 10/IN.1/I3007 and 13/RC/2077. these issues out of the scope of our work, as (i) they are seldom seen in real-world mobile networks, with the partial exception of MNOs blocking peer-to-peer traffic, and (ii) they would conflict with the right here, right now spirit driving users and providers of mobile services.

In our model, OTTs can decide to enter into service level agreements (SLAs) with an MNO to get a certain QoS for their services. In exchange for a fee, the MNO will reserve enough capacity to satisfy the QoS expected. The OTTs would need to decide whether it is more cost effective to rely on SLAs with selected MNOs or to deploy their own network infrastructure. The MNOs, in turn, would factor SLAs with OTTs into their decision of whether and how to expand their networks. In other words, we will enter the age of service-driven network expansion, and, more forwardlooking, service-driven networks.

In order to study service-driven network expansion we need to assess, first, which factors are likely to influence SLAs, and second, the characteristics of the resulting networks. The former are presented on the left hand side of Fig. 1 and include technical and non-technical aspects. Factors considered include the technologies available (e.g. LTE, WiFi) and their costs, public policy and regulation (e.g., whether to release new bands to the public, spectrum licensing schemes), and the characteristics of the demand. The resulting network characteristics are presented on the right hand side of Fig. 1 and include, for example, the level of heterogeneity of the resulting network in terms of both ownership and technology, the use of licensed/unlicensed spectrum, and the emergence of virtual networks tailored to OTTs. The likely result is a move from the current paradigm, where networks are designed, owned and controlled by MNOs, to a new one, where OTTs have a major role in the deployment of new infrastructure. Infrastructure will tend to become more heterogeneous, and integrate different equipment, some of which will operate on unlicensed spectrum (e.g., ISM bands, as in LAA-LTE [7]).

The purpose of this paper is to investigate, qualitatively 


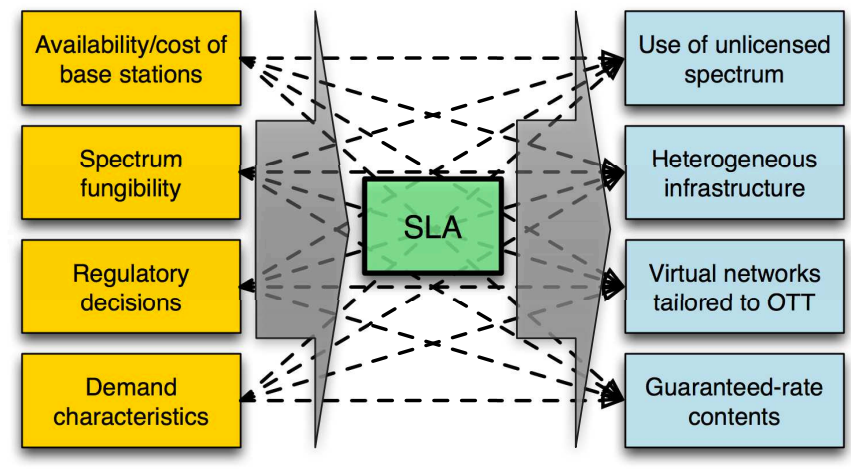

Fig. 1: The features of service-driven networks (blue boxes on the right hand side), and the factors driving them (yellow boxes on the left hand side) map into parameters and decision variables of our system model, respectively. Our high-level goal is to study the relationship between all those quantities, i.e., to untangle the network of arrows between the blocks.

and quantitatively, the impact of the different factors, listed on the left-hand side of Fig. 1, on the SLAs between an $\mathrm{MNO}$ and OTTs and on the planning decisions for servicedriven networks. The first contribution of this work is a synthetic model of a cellular network deployment that accounts for some of the most relevant aspects of the servicedriven network deployment described in Fig. 1 and how they interact with each other. For example, the set of technologies available to OTTs in our model will depend on regulatory decisions, e.g., whether OTTs will be allowed to use licensed spectrum. Model parameters will account for the extent to which different parts of the spectrum can be considered equivalent to each other (e.g., intuitively, how many megahertz of Wi-Fi spectrum are needed to obtain the same performance of one megahertz of LTE spectrum), a concept often called fungibility [8]. Our model captures the decisions made by OTTs about whether and how to deploy their own infrastructure; as an example, we have a decision variable expressing whether each operator deploys a base station of a certain type at a certain location, and parameters expressing the cost of doing so.

After presenting our model in Sec. 2, we detail in Sec. 3 our solution concept, showing how the main actors involved in the network expansion process efficiently make selfinterested, near-optimal decisions. Sec. 4 contains estimates for all the factors we account for. Results, obtained for the real-world topology described in Sec. 5 and summarized in Sec. 6, show the impact of these factors on how and by whom service-driven networks will be built and operated.

\section{System model}

In this section, we present our system model, summarized in Fig. 2. We consider a snapshot of the network, taken during high-load conditions that are typically [9, Sec. 10.3.3.2] used as a reference when planning a network. The purpose of our model is to capture the network conditions in a challenging situation (as detailed in Sec. 4 and Sec. 5), so its infrastructure can be planned accordingly. In this study, we also assume that backhaul is not a limiting factor for network capacity.

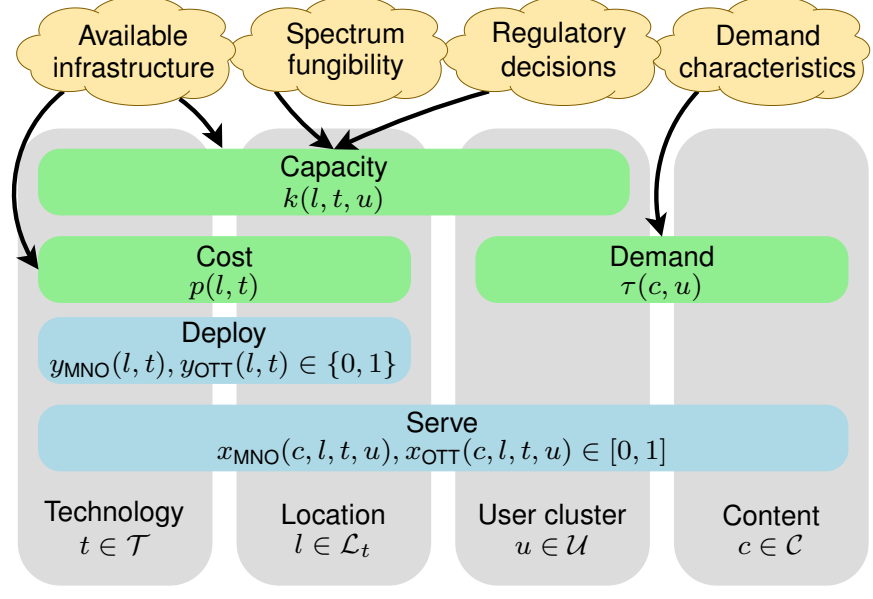

Fig. 2: Our system model. Grey, vertical blocks represent the model entities. Horizontal blocks correspond to parameters (green blocks) and decision variables (blue ones). Horizontal and vertical blocks cross when the parameter/variable represented by the horizontal block is indexed by the entity represented by the vertical one, e.g., a deployment decision is made for each technology and base station. Yellow clouds, corresponding to the boxes on the left hand side of Fig. 1, indicate the sources of our parameters.

The level of abstraction of our model is one of the most critical decisions we have to make. Cellular networks are highly complex entities, and any model trying to capture all this complexity would be exceedingly difficult to handle. As such, we employ simplified models to estimate, for example, the capacity for the different technologies, or the candidate locations of new base stations. However, it is important to stress that our goal is not to propose a comprehensive model for cellular network operations, but rather to study the relationships shown in Fig. 1 that influence network planning decisions of service-driven networks.

Another important decision deals with the level of determinism of our model. Three options were possible:

1) a fully probabilistic model, where we need to know the distribution for all quantities, and are able to estimate the distribution of the objective function and the probability that each constraint be met;

2) a hybrid model, where quantities are known as distributions, but constraints need to be met with a static target probability;

3) a fully deterministic model, where parameters are known as fixed, worst-case values.

We opt for the third possibility, for simplicity and efficiency considerations but most importantly because we are dealing with network planning decisions, which can take years to implement and whose effects can last for decades. These decisions are usually made based on longterm, worst-case projections and forecasts.

In other words, every time we mention, for example, "the user traffic", we actually refer to the worst-case user traffic that can be realistically expected. It follows that the deployments resulting from our model are remarkably resilient to demand and propagation conditions. It also means that they might be slightly more costly, for OTTs and MNOs, 
than they could, but this is consistent with the fact that no network provider is willing to risk service disruptions to their users [10].

\section{System elements}

Our system model includes four elements, represented by grey vertical blocks in Fig. 2: technologies, locations, user clusters and content types. The sets of technologies, locations, clusters and content types are, within our system model, input data. In Sec. 4 and Sec. 5 we will discuss how and where this information is gathered.

Technologies $t \in \mathcal{T}$ represent the available types of network infrastructure. LTE macro and micro base stations as well as WiFi and mmWave access points correspond to different technologies; furthermore, base stations using different frequencies or power levels also correspond to different technologies. In general, if two infrastructure elements have different cost or coverage or performance, then in our model they correspond to different technologies. Some technologies require specific permissions or a license to operate (e.g. LTE base stations in licensed bands) and they are unlikely to be deployed by the OTTs. Therefore, we denote as $\mathcal{T}_{\text {OTT }} \subseteq \mathcal{T}$ the set of technologies available to the OTTs, while $\mathcal{T}_{\text {MNO }} \equiv \mathcal{T}$ corresponds to the technologies available to the MNOs.

Locations $l \in \mathcal{L}_{t}$ represent the positions in space at which infrastructure of technology $t \in \mathcal{T}$ may be located. As an example, each building within an urban area may correspond to a location. In the following, we will often refer to the combination of a location $l \in \mathcal{L}_{t}$ and a base station type $t \in \mathcal{T}$ as a base station.

User clusters $u \in \mathcal{U}$ represent groups of users that can be seen as co-located. Indeed, when performing network planning, we are not interested in the position or mobility of individual users, but rather in the total number of users in a constrained geographic area.

Last, Content types $c \in \mathcal{C}$ (hereinafter "contents") represent the types of content users are interested in accessing such as HD video or on-line gaming.

\section{Parameters}

Parameters are known quantities associated with one or more elements of our system model. They are represented by green horizontal blocks in Fig. 2. From the viewpoint of our model, they are input values; however, as denoted by the yellow clouds in the figure, they actually come from the sources listed in Sec. 4.

The first parameter is the estimated capacity $k(l, t, u)$ that a base station of technology $t \in \mathcal{T}$, built in location $l \in \mathcal{L}_{t}$, would be able to offer to users in cluster $u \in \mathcal{U}$ if it serves no other clusters, and it is based on a simplified model as expressed in:

$$
k(l, t, u)=f(t) B(t) \eta(l, t, u), \quad \forall t \in \mathcal{T}, l \in \mathcal{L}_{t}, u \in \mathcal{U},
$$

where $f(t)$ indicates the performance penalty incurred in when using unlicensed frequencies (see Sec. 4.2), $B(t)$ is the bandwidth available to technology $t$ (see Sec. 4.1), and $\eta(l, t, u)$ is the spectral efficiency that a base station of technology $t$ in location $l$ can deliver to user cluster $u$. The spectral efficiency is estimated using the Shannon bound and accounts for the distance between a location and a user cluster, the propagation model, and the specific technology employed. The maximum spectral efficiency achievable is also limited by the technology considered (see Sec. 4.1) as expressed in Eq. (2):

$$
\eta(l, t, u) \leq \eta_{\max }(t), \quad \forall t \in \mathcal{T}, l \in \mathcal{L}_{t}, u \in \mathcal{U} .
$$

Notice that $\eta$-values can also account for site-specific information, if available - as an example, a site with a commanding view on top of a hill will have a higher efficiency than a tower on a flat ground surrounded by trees. Moreover, $\eta$-values also account for interference. Specifically, interference from legacy, pre-existing deployments is embedded in the initial $\eta$-values, similarly to other sitespecific information. Interference from new deployments, i.e., new base stations deployed by OTTs and MNOs, can be accounted for in a similar way, at the cost of recomputing the $\eta$-values, as discussed in Sec. 3.

We also need to know the cost $p(l, t)$ of building a base station of technology $t \in \mathcal{T}$ in location $l \in \mathcal{L}_{t}$. Cost ranges for different technologies can be extracted from the literature, as detailed in Sec. 4. If such information is available, costs can also incorporate rent and maintenance, and sitespecific features like existing rights-of-way to honor.

Costs $p$ are per-year: they include recurring expenses (e.g., rent and energy) and the amortization of one-time costs, e.g., equipment. This allows our model to describe both green-field scenarios, where networks are built from scratch, and scenarios where network evolves from one generation to the next. In the latter case, existing infrastructure is taken into account by lowering the cost of those $(l, t)$ combinations for which there is a base station of type $t$ already deployed at location $l .^{1}$

Last, we have the demand $\tau(c, u)$ requested by users in cluster $u \in \mathcal{U}$ for content type $c \in \mathcal{C}$. As discussed in Sec. 1, in our model the demand is a given parameter: MNOs and OTTs alike have no way of influencing it, neither through price incentives nor through traffic shaping techniques. As the authors of [11] put it, our users are impatient.

\section{Variables}

Variables correspond to the decisions MNOs or OTTs make. They are represented by blue horizontal boxes in Fig. 2 .

The first task the MNO faces is to propose an SLA fee to the OTTs, where the OTTs has to pay for their contents to be given a guaranteed bitrate. Such a fee may be a direct payment or some other form of revenue transfer from OTTs to the MNOs [?], [6]. In our model, we represent it through a per-megabit fee $\beta$ charged to the OTT to have its traffic served by the MNO's network.

The following decisions concern whether or not the OTTs deploy a base station of technology $t \in \mathcal{T}$ at location $l \in \mathcal{L}_{t}$ represented through a binary variable $y_{\text {Отт }}(l, t)$. Parallel decisions are how to serve the users, i.e., the fraction of the total time and frequency resources (in LTE terminology, physical resource blocks, PRBs) a base station of technology $t \in \mathcal{T}$, deployed at location $l \in \mathcal{L}_{t}$, uses to meet the

1. Though not necessarily setting them to zero, as existing base stations may still be subject to recurring costs. 
demand of a subscriber requesting content $c \in \mathcal{C}$ located in cluster $u \in \mathcal{U}$. This is expressed through a real-valued variable $x_{\text {OTT }}(c, l, t, u) \in[0,1]$. Finally, the $\mathrm{MNO}$ has to deploy its own infrastructure and decide how to serve the residual demand for all the contents. These decisions can be represented by $y_{\mathrm{MNO}}(l, t)$ and $x_{\mathrm{MNO}}(c, l, t, u)$, respectively. $y_{\mathrm{MNO}}$ and $x_{\mathrm{MNO}}$ indicate the same type of decision variables as $y_{\text {OTT }}$ and $x_{\mathrm{OTT}}$, but they refer to the $\mathrm{MNO}$ rather than the OTT.

\section{SOLUTION CONCEPT}

Our model accounts for the two main actors involved in deploying and managing service-driven networks, i.e., traditional MNOs and OTTs. Each actor is self-interested and ultimately aims at maximizing its own profit. In this section, we detail the decision process they take part in, and how individual decisions are made.

\subsection{Decision process}

In our model, both OTTs and the MNO seek to maximize their profit (or, equivalently, minimize their costs, as revenue obtained from end users is assumed constant). Both need to decide what infrastructure of their own to deploy, in which location, and of what type. OTTs also need to decide how much to rely on the MNO to serve their content types, and the MNO needs to decide how much to charge OTTs to satisfy QoS requirements specified in the SLA for the OTTs' contents.

In the first stage, the MNO decides the fee, i.e., the permegabit price that OTTs have to pay if they want their contents to be delivered at a certain bitrate. Fees have an effect on the revenue the MNO collects. Intuitively, setting low fees indicates potentially low revenue for the MNO, which has to serve more traffic (hence update its network) for little additional revenue. On the other hand, setting very high fees represents a stronger incentive for OTTs to deploy their own infrastructure, rather than paying the $\mathrm{MNO}$ for their content to be delivered.

In the second stage, OTTs have to plan their infrastructure. For each part of the topology, they can choose between having the MNO serve their demand therein - and paying the fee - or serving the demand themselves, deploying their own base stations - and bearing the related cost.

In the third stage, MNOs have to make decisions regarding deployment in their network. They know they have to serve all the demand left unserved by the OTTs in order to honor their commitments, by deploying the necessary infrastructure while minimizing their costs.

Note that, at every stage of the solution, we assume that the demand will be served, i.e., that the dimensioning problem is feasible. This assumption reflects the widespread belief that it will be possible for cellular networks to cope with the challenge posed by the increase in data demand, and we seek the best way to do so.

\subsection{Individual steps}

In the following, we detail how the MNOs and the OTTs make their decisions in each step of the process described in Fig. 4, and specifically, the problem they seek to optimize and the method they employ to do it. We start by presenting the deployment decisions for each OTT, then the subsequent deployment decisions for the MNO. We reserve special attention to the problem of setting the fee at the end of this section.

\section{OTT - minimize the costs to serve the demand}

We now focus on the deployment decision problem from the point of view of the OTTs. We assume that each OTT knows the characteristics of its own demand, expressed as $\tau(\hat{c}, u)$, where $\hat{c}$ is the content type that belongs to the OTT considered. The OTT can choose between serving the demand directly, deploying its own infrastructure, or using the MNO's infrastructure and paying the fee $\beta$. We assume that the $\beta$ fee at this stage is known. Each OTT wants to maximize its profit, which is in this case equivalent to minimizing the total cost, i.e.,

$$
\begin{aligned}
& \min _{x_{\mathrm{OTT}}, y_{\text {OTT }}}\left(\beta \sum_{u \in \mathcal{U}} \bar{\tau}(\hat{c}, u)+\sum_{t \in \mathcal{T}_{\text {OTT }}} \sum_{l \in \mathcal{L}_{t}} y_{\mathrm{OTT}}(l, t) p(l, t)\right) \\
& \sum_{t \in \mathcal{T}_{\text {OTT }}} \sum_{l \in \mathcal{L}_{t}} x_{\text {OTT }}(\hat{c}, l, t, u) k(l, t, u) \leq \tau(\hat{c}, u), \quad \forall u \in \mathcal{U} \\
& \sum_{u \in \mathcal{U}} x_{\text {OTT }}(\hat{c}, l, t, u) \leq y_{\mathrm{OTT}}(l, t), \quad \forall t \in \mathcal{T}_{\mathrm{OTT}}, l \in \mathcal{L}_{t},
\end{aligned}
$$

where $\bar{\tau}$ is the residual demand, expressed as the difference between the total demand and the demand that is served by the OTT's base stations:

$$
\bar{\tau}(\hat{c}, u)=\tau(\hat{c}, u)-\sum_{t \in \mathcal{T}_{\mathrm{OTT}}} \sum_{l \in \mathcal{L}_{t}} x_{\mathrm{OTT}}(\hat{c}, l, t, u) k(l, t, u) .
$$

The first term in Eq. (3) represents the fees paid to the MNO to serve the residual demand. The second term is the cost incurred by the OTT to deploy its own infrastructure. The OTT has no constraints to serve all its demand itself as indicated in Eq. (4): any residual demand $\bar{\tau}$ will be served by the MNO, as described earlier, in exchange for a fee. Moreover, constraint Eq. (4) indicates that the OTT does not serve more traffic demand then it has to, thus it prevents the quantity $\bar{\tau}(\hat{c}, u)$ from taking negative values. Eq. (5) ensures that we properly account for the maximum

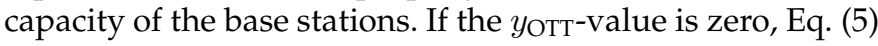
indicates that the base station cannot serve any user at all. If it is one, it prevents base stations from serving more traffic than they can, i.e., more than their capacity. The OTT is concerned with minimizing its total cost expressed in Eq. (3); if building no base station at all serves such a purpose, there is nothing forcing OTTs to do otherwise.

It is worth observing that the residual demand $\bar{\tau}$ is a decision variable for the OTTs, and, after we obtain the $\bar{\tau}$ for all the content types $c \in \mathcal{C}$, it becomes an input parameter in the MNO deployment problem we examine in the next section.

\section{MNO - minimize the deployment costs}

At this stage, the MNO obtains the residual demand $\bar{\tau}(c, u)$ for each user cluster and content type, i.e., the demand that the OTTs decided to have served by the MNO, in exchange 
for the fee $\beta$. The MNO has to deploy infrastructure, i.e., setting $y_{\mathrm{MNO}}$-values to one, in order to serve the residual demand. It seeks to satisfy the demand with minimum costs:

$$
\begin{gathered}
\min _{x_{\mathrm{MNO}}, y_{\mathrm{MNO}}} \sum_{t \in \mathcal{T}_{\mathrm{MNO}}} \sum_{l \in \mathcal{L}_{t}} y_{\mathrm{MNO}}(l, t) p(l, t) . \\
\sum_{t \in \mathcal{T}_{\mathrm{MNO}}} \sum_{l \in \mathcal{L}_{t}} x_{\mathrm{MNO}}(c, l, t, u) k(l, t, u) \geq \bar{\tau}(c, u), \quad \forall c \in \mathcal{C}, u \in \mathcal{U}
\end{gathered}
$$

$$
\sum_{u \in \mathcal{U}} \sum_{c \in \mathcal{C}} x_{\mathrm{MNO}}(c, l, t, u) \leq y_{\mathrm{MNO}}(l, t), \quad \forall t \in \mathcal{T}_{\mathrm{MNO}}, l \in \mathcal{L}_{t}
$$

Eq. (7) has to satisfy the constraints on the traffic demand and on the capacity expressed by Eq. (8) and Eq. (9). The constraint expressed by Eq. (8) ensures that the MNO provides enough capacity for all user clusters $u \in \mathcal{U}$ and contents $c \in \mathcal{C}$ the MNO must serve, while the constraint in Eq. (9) ensures that we properly account for the maximum capacity of the base stations and that only active base stations are used.

We note that the fee $\beta$ does not appear in the MNO deployment problem - as at this stage, the MNO has already established the fee, and has to serve all the demand the OTT decides to delegate to it.

\section{MNO - maximize the revenue by setting the fee $\beta$}

Setting the fee is the most complex task. It is a decision made by the MNO that depends on the response of the OTTs. The objective of the MNO is maximizing its own profit as shown in the following formula:

$$
\max _{\beta}\left(\beta \sum_{u \in \mathcal{U}} \sum_{c \in \mathcal{C}} \bar{\tau}(c, u)-\sum_{t \in \mathcal{T}_{\mathrm{MNO}}} \sum_{l \in \mathcal{L}_{t}} y_{\mathrm{MNO}}(l, t) p(l, t)\right)
$$

The objective function expressed in Eq. (10) is composed by two terms. The first indicates the revenue that the MNO collects by serving the residual demand for each content type that belongs to the OTTs. The second term is the cost incurred in by the MNO to deploy additional infrastructure to serve the residual demand. The residual demand appears twice in this optimization problem, explicitly in the objective function in the first term, and implicitly in the second term in the form of the constraint in Eq. (8).

The residual demand $\bar{\tau}(c, u)$ depends upon decisions made by the OTTs that are affected by the fee $\beta$, as we have seen previously. It is clear at this stage that the problem of optimizing the fees by the MNO is entwined with the problem faced by the OTTs to minimize their own costs. To circumvent this issue, we depict a strategy that we illustrate in Fig. 3.

At first, the MNO observes the traffic demand and infers the characteristics of the demand. The MNO then tries to optimize the fees by evaluating its utility function expressed in Eq. (10) for several values of $\beta$ and then selecting the best one. The MNO essentially has to solve a univariate discrete optimization problem that now involves an iterative process. The MNO estimates the residual demand left unserved

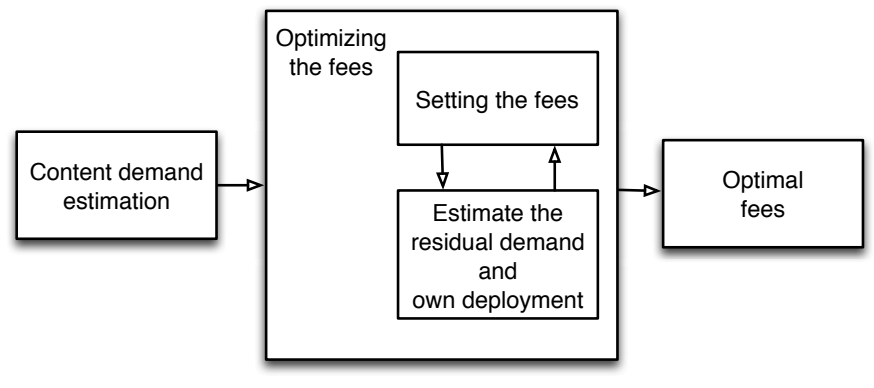

Fig. 3: Strategy to set the optimal fees from the MNO perspective. The MNO has to estimate the action of each OTT, assuming they seek to minimize their own cost.

by each OTT and subsequently the new infrastructure necessary to serve the whole residual demand for each value of $\beta$ by sequentially solving both the OTT's deployment problem and its own.

Each fee's configuration in fact yields a different residual demand $\bar{\tau}$. However, the final network deployment corresponds to the one resulting from the optimum $\beta$ from the perspective of the MNO.

\subsection{Solution strategy}

In the following, we discuss how each of the problems presented above can be solved.

The OTTs and MNO deployments, corresponding to the internal boxes in Fig. 4, have the same structure. They are linear problems with integer (binary) and real variables, so they belong to the mixed integer linear programming (MILP) class; they can be solved to optimality with branchand-cut algorithms using commercial state-of-the-art solvers such as Gurobi or CPLEX, provided that the size of the problem is not too large. If the size of the problem is too large, we would face the well-known scalability issues of MILP problems. In this case, we would turn to heuristic solutions such as the ones described in [12], originally formulated for set-covering problems. Their greedy approach has been shown, through extensive studies carried out on the OR problem library [13], to consistently perform close to the optimum, with a ratio between the solution they find and the optimal one being typically around 1.2.

The problem solved by the MNO when setting the fee $\beta$ is even more challenging, lacking a closed-form expression. However, in light of our strategy described in Fig. 3, the new problem becomes a univariate problem in $\beta$, and therefore can be solved heuristically with root-finding algorithms such as the Brent method [14].

Root-finding algorithms explore several possible values of the decision variable, evaluate the value of the objective function, and use such information to select the next values to try. In our case, each iteration of the Brent method solves the problems Eq. (3) and Eq. (7) in sequence; their outputs are used to compute the payoff Eq. (10) and to find the optimal value of $\beta$.

If we decide that spectral efficiency values $\eta$ also need to account for interference from new deployments, they would need to be recomputed at every iteration of the Brent method, as they would depend upon decisions made 
therein. $^{2}$ Also notice that in practice, when deploying additional infrastructure, MNOs and OTTs can account for the resulting change in the interference environment through frequency planning and power control.

The scalability of the overall solution concept is ensured by the fact that the Brent method requires a limited number of iterations to converge, and each iteration takes a limited amount of time to solve subproblems Eq. (3) and Eq. (7).

Although they have been consistently observed to perform very well in practice, neither the Brent method nor the heuristic in [12] come with a formal, absolute optimality guarantee. This is indeed consistent with our goals: we seek to confirm and uncover correlations between the conditions under which service-driven networks will operate and the features they will exhibit; to this end, heuristic solutions are essentially as useful as optimal ones.

Another relevant feature of our solution strategy is that it uses off-the-shelf components whenever available, either commercial solvers like CPLEX and Gurobi or wellestablished software like MATLAB and NumPy. Doing so allows us to focus on the solution strategy and the results it yields, as opposed to fine-tuning its building blocks. Furthermore, it provides us with the efficiency we need to process our datasets.

\section{FACTORS SHAPING SERVICE-DRIVEN MOBILE NETWORKS}

In this section, we describe the factors that will drive and shape service-driven mobile networks: the availability of new types of base stations; the fungibility of different portions of the spectrum; the regulatory decisions constraining the deployment of network infrastructure; the demand it will need to serve.

For each of these elements, we explain how it is captured within the model described in Sec. 2. We then review the estimates for its value existing in the literature, and determine either a value or a range of values to use in our performance evaluation.

\subsection{Base station technologies}

Heterogeneity will be an important feature of service-driven mobile networks. Different types of base stations will coexist therein, including:

- $\quad$ LTE macro-base stations (macroBSs);

- LTE micro-base stations (microBSs);

- millimeter-wave base stations (mmWave);

- Wi-Fi access points.

Additional types of base stations can be added to $\mathcal{T}$ as sufficiently detailed information about them becomes available such as Google's Project Loon [15], LTE balloonpowered platforms operating in unlicensed bands used to provide LTE coverage to rural areas, or Facebook's Connectivity Project [?].

Infrastructure types that operate in licensed bands (hence with exclusive usage rights) can only be deployed

2. Refreshing the $\eta$-values would marginally improve the accuracy of the results yielded by this model, at the cost of a substantially higher computational complexity. For this reason, we leave $\eta$-values constant for our performance evaluation (Sec. 6). by mobile network operators (e.g., LTE macroBSs and LTE microBSs at $1.8 \mathrm{GHz}$ and $2.6 \mathrm{GHz}$ ), while technologies operating in shared bands can be deployed by both MNO and OTT (e.g., mmWave, LTE microBSs at 3.5GHz, Wi-Fi) having different costs and fungibility (see Sec. 4.2).

Tab. 1 summarizes the types of infrastructure we consider in this study, i.e., the elements of set $\mathcal{T}$. For each of them, we indicate the frequency they operate at, their bandwidth, their maximum spectral efficiency, and the resulting maximum capacity - this is an upper bound on the values of parameter $k(l, t, u)$. Notice that microBSs with exclusive and opportunistic access are considered as two separate elements of $\mathcal{T}$.

\subsubsection{Cost}

Estimating the cost of a base station is a difficult exercise. We gathered the figures indicated in Tab. 1 from peerreviewed publications where available, and falling back to other sources such as business/technical reports when needed. Despite extensive research, we were unable to find a single cost estimate for mmWave base stations, other than generic claims that they will be inexpensive. We conjecture that their cost will lie between the most expensive Wi-Fi access points and the cheapest microBSs.

Recall that, as mentioned in Sec. 2, our costs $p(l, t)$ are per-year. It follows that the values in Tab. 1 include both one-time costs (e.g., infrastructure) and recurring costs (e.g., energy or rent). Also, note that, for simplicity, we assume the price for each base station of technology $t \in \mathcal{T}$ to be the same regardless of the location $l \in \mathcal{L}_{t}$.

\subsubsection{Performance}

Our system model (Sec. 2) includes a parameter $\eta(l, t, u) \leq$ $\eta_{\text {max }}(t)$ describing the actual spectral efficiency attained by a base station of technology $t$ deployed at location $l$ when serving users in cluster $u$. The main factors influencing $\eta$ values are path-loss and interference, which we estimate through ITU- and 3GPP-vetted propagation models [22], [23].

Fig. 5 shows the distribution of the $\frac{\eta(l, t, u)}{\eta_{\max }(t)}$ ratio for the different technologies, throughout all the experiments we run in our analysis. We can observe that technologies operating on licensed frequencies, e.g., macroBSs, tend to have a better ratio - even though the median macroBS has a spectral efficiency that barely exceeds $2 \mathrm{bps} / \mathrm{Hz}$. mmWave and Wi-Fi never go beyond $50 \%$ and $70 \%$ of their potential efficiency.

\subsection{Spectrum fungibility}

Fungibility as a concept has roots in the economic process of trade. The core of this concept is the ability to substitute one item with another, without altering any other aspect of a trade. As such, this concept implies two distinct roles, a buyer and a seller, with the former having the power to declare items fungible. In terms of spectrum, the buyer may be a network operator and the seller may be a regulatory agency, which offers usage of a particular set of frequencies. Two bands of spectrum are considered fungible if the operator is happy to obtain either one at a given time and price. 


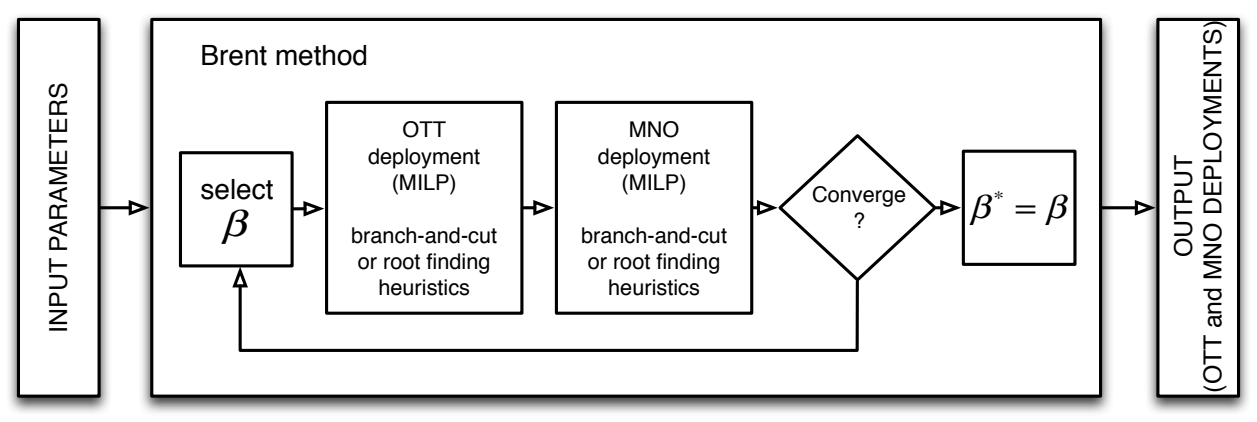

Fig. 4: Solution strategy implementation overview.

TABLE 1: Infrastructure types populating set $\mathcal{T}$.

\begin{tabular}{|c|c|c|c|c|c|c|c|}
\hline $\begin{array}{c}\text { Base station } \\
\text { technology }\end{array}$ & Frequency & $\begin{array}{c}\text { Bandwidth } \\
B(t)\end{array}$ & $\begin{array}{c}\text { Max. spectral } \\
\text { efficiency } \eta_{\max }(t)\end{array}$ & $\begin{array}{c}\text { Max. } \\
\text { capacity }\end{array}$ & $\begin{array}{c}\text { Tx power } \\
\text { Cost range }\end{array}$ & $\begin{array}{c}\text { Approx. } \\
\text { range }\end{array}$ \\
\hline \hline LTE macroBS & $\begin{array}{c}1.8 \mathrm{GHz} \\
\text { (licensed) }\end{array}$ & $20 \mathrm{MHz}$ & $\begin{array}{c}4.4 \mathrm{bps} / \mathrm{Hz} \\
{[16]}\end{array}$ & $88 \mathrm{Mbps}$ & $40 \mathrm{dBm}$ & $\begin{array}{c}{[10000,60000]} \\
€ / \text { year [17], [18] }\end{array}$ & $\begin{array}{c}\text { Several hundreds } \\
\text { of meters }\end{array}$ \\
\hline LTE microBS-L & $\begin{array}{c}2.6 \mathrm{GHz} \\
\text { (licensed) }\end{array}$ & $20 \mathrm{MHz}$ & $\begin{array}{c}4.4 \mathrm{bps} / \mathrm{Hz} \\
{[16]}\end{array}$ & $88 \mathrm{Mbps}$ & $33 \mathrm{dBm}$ & $\begin{array}{c}{[2000,10000]} \\
€ / \text { year [17], [18] }\end{array}$ & $\begin{array}{c}\text { Few hundreds } \\
\text { of meters }\end{array}$ \\
\hline LTE microBS-S & $\begin{array}{c}3.5 \mathrm{GHz} \\
\text { (shared) }\end{array}$ & $20 \mathrm{MHz}$ & $\begin{array}{c}4.4 \mathrm{bps} / \mathrm{Hz} \\
{[16]}\end{array}$ & $\begin{array}{c}<88 \mathrm{Mbps} \\
\text { (fungibility }<1)\end{array}$ & $33 \mathrm{dBm}$ & $\begin{array}{c}{[2000,10000]} \\
€ / \text { year [17], [18] }\end{array}$ & $\begin{array}{c}\text { Few hundreds } \\
\text { of meters }\end{array}$ \\
\hline $\begin{array}{c}\text { mmWave } \\
{[19],[20]}\end{array}$ & $\begin{array}{c}73 \mathrm{GHz} \\
\text { (shared) }\end{array}$ & $500 \mathrm{MHz}$ & $\begin{array}{c}2.25 \mathrm{bps} / \mathrm{Hz} \\
\text { (fungibility }<1)\end{array}$ & $30 \mathrm{dBm}$ & $\begin{array}{c}<1125 \mathrm{Mbps} \\
€ / \text { year }\end{array}$ & $\begin{array}{c}\text { Tens of } \\
\text { meters }\end{array}$ \\
\hline Wi-Fi & $\begin{array}{c}2.4,5 \mathrm{GHz} \\
\text { (shared) }\end{array}$ & $20 \mathrm{MHz}$ & $\begin{array}{c}3.12 \mathrm{bps} / \mathrm{Hz} \\
{[21]}\end{array}$ & $\begin{array}{c}<62.4 \mathrm{Mbps} \\
\text { (fungibility }<1)\end{array}$ & $24 \mathrm{dBm}$ & $\begin{array}{c}1000 \\
€ / \text { year [17], [18] }\end{array}$ \\
\hline
\end{tabular}

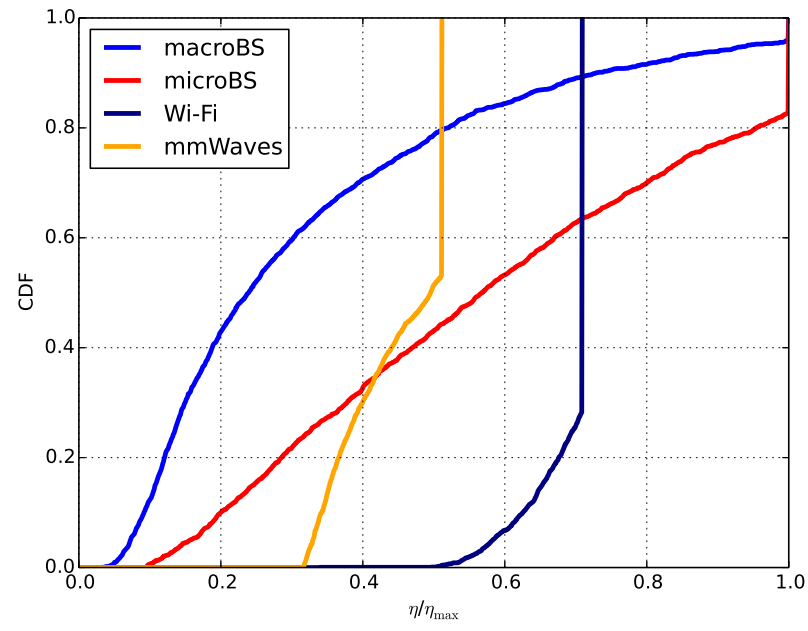

Fig. 5: Distribution of the ratio between the actual spectral efficiency values $\eta(l, t, u)$ and the corresponding upperbounds $\eta_{\max }(t)$, for different technologies.

As spectrum usage becomes more fluid, the concept of fungibility of spectrum becomes increasingly important [8]. Network operators have the ability to divert traffic between a number of different bands and technologies, buying this capacity on demand; fungibility provides an important tool to assess the relative usefulness of these options based on the goals of the operator.

Different portions of spectrum are not fungible, for three reasons:

- different frequencies are associated with different bitrate and coverage;

- different frequencies typically require different hardware running different protocols;
- unlicensed frequency bands are more prone to congestion and interference than licensed ones.

From the point of view of our model, all three aspects above are embedded in the fungibility parameter $f(t)$ which we use to estimate the capacity $k(l, t, u)$, as discussed in Sec. 4.1. Fungibility is a coefficient by which we further multiply the capacity as listed in Tab. 1; its values are presented in Tab. 2.

TABLE 2: Fungibility coefficients.

\begin{tabular}{|c|c|c|}
\hline Technology & $\begin{array}{c}\text { Fungibility } f(t) \\
\text { range }\end{array}$ & Source \\
\hline \hline $\begin{array}{c}\text { Small cells } \\
\text { (WiFi and mmWave) }\end{array}$ & $.6-.9$ & {$[24]$} \\
\hline $\begin{array}{c}\text { MicroBSs } \\
\text { (in shared bands) }\end{array}$ & $.5-.75$ & {$[25]$} \\
\hline
\end{tabular}

\subsection{Regulatory decisions}

Licensed spectrum is a scarce resource. Hence, who will be allowed to use it, and how, have a critical impact on network performance, and heavily depend on regulatory decisions. In our work, we examine two scenarios concerning which portions of the spectrum, and under which conditions, OTTs have access to:

- LTE-standard complying base stations can only transmit in licensed spectrum. OTTs cannot operate microBSs in shared bands [Scenario A].

- LTE-standard complying base stations can be deployed in both licensed and shared spectrum. The OTT and MNO can deploy microBSs, but in shared bands they experience a fungibility coefficient lower than one, as reported in Tab. 2 [Scenario B].

Selecting one or the other of these scenarios changes the elements of set $\mathcal{T}_{\text {OTT, }}$ as well as the adjusted capacity $k$ of base stations. 


\subsection{Demand}

The total demand and set $\mathcal{C}$ of content types depend on the reference scenario we consider, as discussed later in Sec. 5. We seek to study the impact that spatial clustering of intensities for a particular content, i.e., whether users requesting a particular content tend to be close to each other or not, has on service-driven networks. We quantify this clustering through the Hegyi index [26], used to express the clustering strength between spatially distributed points (user clusters, in our case) associated to a continuous value (contents, in our case). The Hegyi index for user cluster $u$ and content $c$ is:

$$
H(c, u)=\sum_{i=1}^{N} \frac{\tau(c, u)}{\tau(c, u)\left(1+\left\|u+n_{i}(u)\right\|\right)},
$$

where $\|\cdot\|$ denotes the Euclidean distance between two user clusters, $n_{i}(u)$ denotes the $i$-th closest cluster to $u$, and $N$ is a parameter denoting the number of nearest user clusters we account for (in our case, $N=5$ ). The complementarity value associated with a specific content type $c^{\prime} \in \mathcal{C}$ is defined as the average over all user clusters $u \in \mathcal{U}$ of $H\left(c^{\prime}, u\right)$ :

$$
H\left(c^{\prime}\right)=\frac{1}{|\mathcal{U}|} \sum_{u \in \mathcal{U}} H\left(c^{\prime}, u\right)
$$

The complementarity value defined in Eq. (12) ultimately tells us how spatially-clustered the demand for a certain content type tends to be. Intuitively, we can expect that a more clustered demand is easier to serve through such targeted infrastructure as the one that can be deployed by OTTs. Location-specific services, e.g., tourist information [27] or advertisements for local businesses [28], are often mentioned as prime examples of content associated to high complementarity values. Other such contents include maps, especially for mobile and vehicular users [29], and, more recently, augmented-reality games such as Pokemon Go [30].

We investigate the correlation between the complementarity of the demand for a particular content with the traits of the resulting network in Sec. 6. Notice that we do not assume a certain complementarity value for our study; rather, we explore what happens if high-complementarity, location-based services come to pass.

\section{Reference scenario}

In this section, we describe the reference scenario we employ for our simulations, i.e., how we populate the sets of user clusters $\mathcal{U}$ and (potential) base station locations $\mathcal{L}_{t}$ for each technology $t \in \mathcal{T}$, and how we set the demand $\tau(c, u)$ for each user cluster and content type.

User clusters and locations: our reference topology is the entire urban area of Dublin, Ireland, as indicated by the Central Statistics Office (CSO) Ireland in [31]. We place a total of 2,210 user clusters throughout this area, such that each cluster represents a population of at most 300 people. It follows that more populated areas tend to have more user clusters; this enables us to study dense deployments in such areas while keeping the overall complexity low. We also assume that the LTE macroBSs deployment is already in place to ensure full coverage and mobility and it is given by the available on-line data [32]. In fact, it makes sense for the MNOs to consider in network planning problems the infrastructure already deployed, especially the costly one.

The possible locations in $\mathcal{L}_{t}$ for technology $t \in \mathcal{T}$ are placed on a regular grid with the inter-site distance depending on the coverage range. For example, the intersite distance for LTE microBS is 100 meters, while for WiFi and mmWave is 50 meters.

Service providers demand and initial deployment: setting the demand values $\tau(c, u)$ is a complex task, for which little information is available and some speculation is unavoidable. We proceed as follows:

1) we set the total demand for each user cluster $u$, i.e., $\sum_{c \in \mathcal{C}} \tau(c, u)$

2) we decide how this total demand is split between contents;

3) we adjust the resulting demand complementarity.

We accomplish the first step by leveraging a set of realworld call-detail record (CDR) information from an Irish mobile operator, referring to a period of two weeks in 2013. We then augment that total demand according to the projections of the Cisco Virtual Network Index [33], and obtain an estimate for mobile data demand over the next 5 years.

Breaking down such an aggregated demand into individual demand for each content is another complex problem. We turn to the measurement work [34], which identifies four traffic patterns, i.e., sets of content types, that users in each location were found to conform to. We assume then that a particular content type can be associated with an OTT.

We randomly associate one traffic profile to each user cluster, adjusting the average distance between two user clusters belonging to the same profile. Intuitively, a small distance means that users wanting the same content types tend to be located close together, hence a higher complementarity as defined in Eq. (12).

Finally, we select the most popular of contents types as $\hat{c} \in \mathcal{C}$, i.e., the demand volume that the OTT has to serve (either through the MNO's network or its own). All other content types are assumed to belong to the MNO.

\section{Results}

In this section we analyze the relationship between the parameters of our model and the resulting base station deployments. Intuitively, we try different combinations of input parameters (the yellow boxes in Fig. 1, e.g., different levels of cost for macroBSs), and observe how and how much they impact the resulting network deployment (the blue boxes in Fig. 1, e.g., how many base stations are deployed and by whom).

As summarized in Fig. 6, we split our analysis into two parts. First, in Sec. 6.1, we seek to understand which input parameters have the deepest influence on the fee $\beta^{\star}$ that the MNO will charge to the OTT for using its network; then, in Sec. 6.2, we assess how $\beta^{\star}$ influences the network deployment.

We focus on $\beta^{\star}$ because this quantity represents the way the two actors we study, namely, MNO and OTT, interact with each other. Doing so also allows us to simplify the 


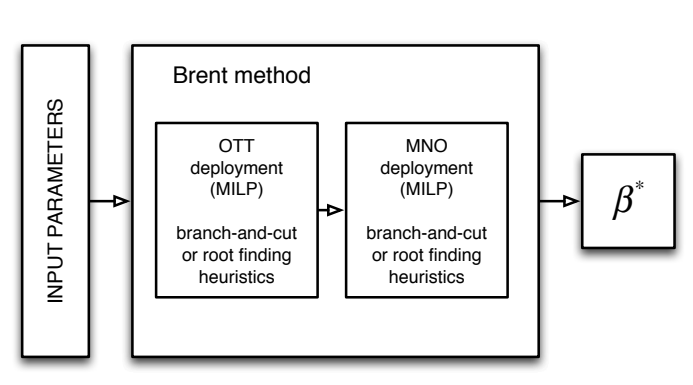

(a)

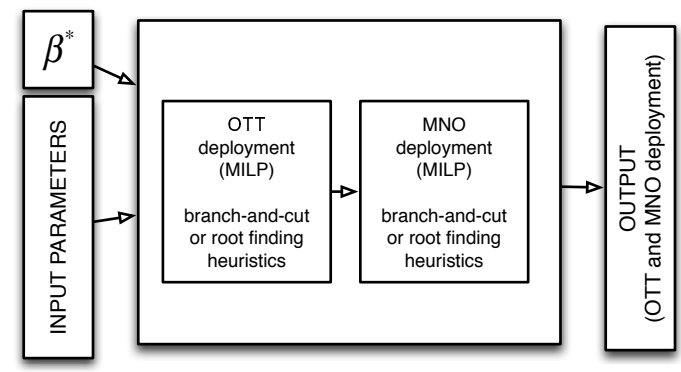

(b)

Fig. 6: Analysis methodology. Once the process in Fig. 4 is completed, we analyse the impact of the input parameters by looking at the problem from two different angles. In (a), we check the influence of the input parameters on the price imposed by the MNO on the OTT $\left(\beta^{\star}\right)$. In (b), we seek to find out the influence of the same input parameters and the $\beta^{\star}$ (now considered as an input parameter) on the output deployment.

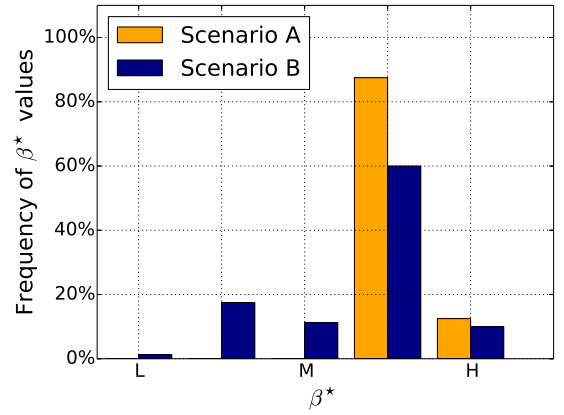

(a)

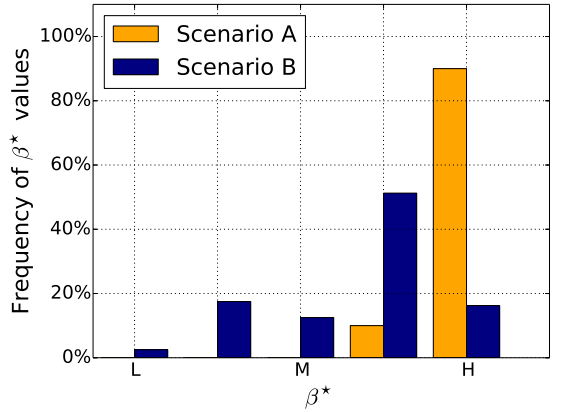

(b)

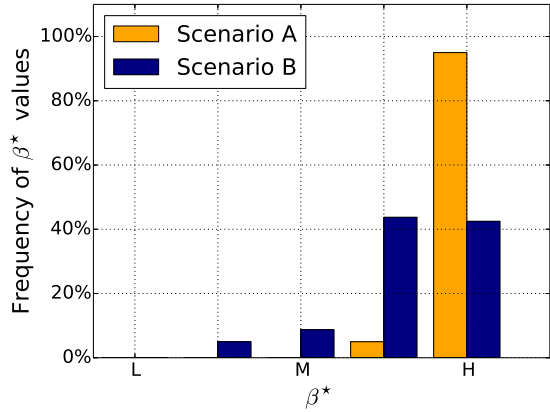

(c)

Fig. 7: Distribution of the fee $\beta^{\star}$ for low (a), medium (b), high (c) complementarity. Bars represent the fraction of $\beta^{\star}$ values that are low, medium, high.

network of relationships shown in Fig. 1 and study, in its stead, only those involving $\beta^{\star}$ itself.

\subsection{What determines $\beta^{\star}$ ?}

The first part of the analysis, as indicated in Fig. 6(a), seeks to assess the key determinants on the variations of $\beta^{\star}$. We carry out a sensitivity analysis employing a multivariable ordinary least-squared (OLS) regression model, where the independent variables are all the ones on the left hand side of Fig. 1 and the dependent variable is $\beta^{\star}$. The total number of parameter combinations we study is 720 .

The results of the regression model are summarized in Tab. 3. As per Fig. 6(a), in this part of our analysis $\beta^{\star}$ is the dependent variable and all other quantities - regulator decisions, demand complementarity, costs, fungibility values - are independent variables. We can immediately notice that among the input parameters chosen to analyse $\beta^{\star}$, only three are actually statistically significant at a pvalue of .01. First, both the regulator and the cost of LTE microBSs have a negative impact on the fee charged by the MNO. The (binary) ${ }^{3}$ variable modelling the regulator allows the OTT access to deploy LTE microBSs in shared spectrum. Infrastructures with wider coverage appear to be very appealing to OTTs since they allow the OTTs to

3. We remark that, while OLS analysis for generic categorical variables requires such special techniques as dummy variables or one-hot encoding, binary independent variables can be used as they are. serve lower-demand subscribers with less infrastructure. As a consequence, the MNO tries to discourage the OTT from deploying many LTE microBS by setting a lower price on the OTT traffic it can serve. Second, the demand complementarity has a non-obvious positive impact on the $\beta^{\star}$. When the majority of the demand is clustered, it can be served with fewer base stations. As a result, the MNO's best action is to set a high fee so as to ensure some gain at least in those regions where the OTT does not find it convenient to deploy any infrastructure. This dual effect is also captured by the histograms in Fig. 7. Preventing OTTs from deploying microBSs, i.e., moving from scenario B to scenario A as defined in Sec. 4.3, generally yields fewer values in the lower bins and more in the higher ones, i.e., increasing the fee intuitively, reducing the freedom of action of OTTs increases the fee they are required to pay.

For completeness, we also report in Tab. 3 the coefficient of determination, $R^{2}$. It measures how well the model captures the variation of the dependent variable [35] and it is defined as:

$$
R^{2}=1-\frac{\sum_{i}\left(s_{i}-\bar{s}\right)^{2}}{\sum_{i}\left(s_{i}-f_{i}\right)^{2}}
$$

where $s_{i}$ and $\bar{s}$ are the sample value and the sample mean respectively, and $f_{i}$ is the modeled/fitted value. 
TABLE 3: Standardized regression analysis coefficients, relative to the first part of our analysis (Fig. 6(a)), where $\beta^{\star}$ is the dependent variable and the quantities listed in the first column are independent variables. The coefficent of determination is $R^{2}=.60$. (see Fig. 6(a)).

\begin{tabular}{|c|c|c|c|}
\hline $\begin{array}{c}\text { Independent } \\
\text { variables }\end{array}$ & coef. $^{*}$ & p-val. $^{* *}$ & std. error \\
\hline \hline Regulator & -.55 & $\checkmark$ & .03 \\
\hline $\begin{array}{c}\text { Demand } \\
\text { complementarity }\end{array}$ & .28 & $\checkmark$ & .02 \\
\hline $\begin{array}{c}\text { Cost } \\
\text { (mmwave) }\end{array}$ & -.00 & $\boldsymbol{X}$ & .09 \\
\hline $\begin{array}{c}\text { Cost } \\
\text { (micro LTE) }\end{array}$ & .40 & $\checkmark$ & .04 \\
\hline $\begin{array}{c}\text { Fungibility } \\
\text { (small cells) }\end{array}$ & .01 & $\boldsymbol{X}$ & .10 \\
\hline $\begin{array}{c}\text { Fungibility } \\
\text { (micro LTE (S)) }\end{array}$ & .00 & $\boldsymbol{X}$ & .12 \\
\hline
\end{tabular}

* It represents the mean change in the dependent variable for 1 unit change in the corresponding independent variable while holding the other independent variables to their mean.

** $\checkmark$ indicates statistical significance (p-value $<$ $.01)$ while $\boldsymbol{X}$ indicates not statistically significant (p-value $>.01)$.

\subsection{What influences the deployment?}

In the second part of the analysis, we focus on the network deployment. We identify, for each operator (i.e., MNO and OTT) and each technology (e.g. WiFi, mmWave, and LTE micro BSs) three parameters of interest: amount of infrastructure built, the capacity supplied and the traffic served by such infrastructure.

There are two main differences from the analysis in Sec. 6.1: first, the $\beta^{\star}$ identified in Sec. 6.1 takes now a much more important role. Together with the original input parameters, we investigate the impact of the $\beta^{\star}$ variable on the deployment, in particular the decision the OTT of whether or not to deploy infrastructure to serve its own demand. To carry out this study we run multiple multivariable OLS regressions, focusing on one outcome variable at a time.

In Tab. 4 we summarize the results obtained by checking one dependent variable at a time. Tab. 4 should be read row-wise, where each row reports the coefficients and $\mathrm{p}$-value of an individual multivariable regression model on the corresponding network deployment output. Tab. 4 lists network deployment quantities (i.e., number of base stations, capacity, traffic served, and level of clustering) grouped by operators and technology in the first column. Remaining columns represent independent variables (i.e., $\beta^{\star}$, regulatory decisions, demand characteristics, technologies cost, fungibility), with the final column reporting the $R^{2}$ value.

A few observations are noteworthy. First, only the cost for LTE microBSs is significant to all network planning actions. Second, the upper part of Tab. 4 reveals that, for the OTT, the $\beta^{\star}$ and the regulatory decisions are influential parameters. They affect all the deployment decisions taken by the OTT, and, to a different extent, the MNO, i.e., defining the heterogeneity of the number of base stations and capacity deployed. The demand complementarity mainly impacts the planning decisions of the OTT, while the decisions taken by the MNO are mostly driven by infrastructure cost.

\subsubsection{Infrastructure cost}

As we have seen in Tab. 4, infrastructure cost has a significant influence on the deployment decisions made by operators and service providers. In Fig. 8(a) we show the number of base stations of each type deployed by the OTT and the MNO. On the $x$-axis is the cost (relative to the $\mathrm{WiFi}$ ) to deploy and maintain a mmWave or an LTE microBS. OTTs are allowed to deploy LTE microBSs in opportunistic access spectrum, i.e., we are in scenario B as described in Sec. 4.3.

Let us compare the group of bars on the left hand side of Fig. 8(a), indicating low costs for infrastructure, with the group of bars in the middle and the right hand side of Fig. 8(a) indicating medium and high costs respectively: if infrastructure is sufficiently cheap, the best course of action for the MNO and OTT is to rely more on LTE microBSs, the ones that give the best compromise between coverage and capacity. As the infrastructure cost increases, both MNO and OTT rely more on WiFi infrastructure. Fig. 8(b) shows how the capacity evolves according to changes in the costs. Highcapacity/short-range technologies will only be successful if their cost is low enough; otherwise, due to their low coverage range, they are unlikely to be deployed. Fig. 8(b) and Fig. 8(c) display an interesting effect: higher capacity does not necessarily translate into more traffic being served if the capacity is very localized, as it is the case with shortrange infrastructure. In fact, as the cost of infrastructure increases, the OTT relies more on the MNO to serve its demand, as it again can be observed in Fig. 8(c).

Fig. 9 and Fig. 10 provide a closer look at how the combination of prices for microBSs and mmWave influence the decisions made by the OTT and MNO. Fig. 9(a) and Fig. 10(a) show that OTTs essentially choose between microBSs and Wi-Fi access points, while they resort to mmWave base stations only when their cost is low. Consistently with Fig. 8(b), Fig. 9(b) and Fig. 10(b) show that mmWave base stations skew the network capacity, even when, as we can see in Fig. 8(c), Fig. 9(c) and Fig. 10(c), microBSs serve most of the traffic.

Service-driven networks will be different from current ones in that the network technology providing the most capacity may not be the one serving the most traffic. This leaves room for innovative applications e.g., proximity services and machine-to-machine systems - as long as they do not require ubiquitous, continuous coverage.

\subsubsection{Demand complementarity}

As seen in Sec. 6.1 and in particular in Fig. 7, the demand complementarity has a non-negligible impact on the fee $\beta^{\star}$ that the MNO charges the OTT to carry its traffic, i.e. the $\beta$ value that maximizes the quantity in Eq. (10).

Complementarity also affects the extent to which the network deployments of the MNO and, especially, of the OTT follow the demand. Fig. 11 refers to the scenario with medium infrastructure prices and high complementarity. We can see from Fig. 11(a) that the demand for content $\hat{c}$ tends to be clustered. Fig. 11(b), showing the capacity deployed by the OTT, clearly follows the same pattern - the OTT deploys more capacity where it has more demand. The first thing we can observe by looking at Fig. 11(c), depicting the capacity deployed by the MNO, is that it does not clearly follow the 
TABLE 4: Standardized regression analysis coefficients, p-values and $R^{2}$ for the system in Fig. $6(\mathrm{~b})$.

\begin{tabular}{|c|c|c|c|c|c|c|c|c|c|c|c|c|c|c|c|c|c|}
\hline \multirow{2}{*}{\multicolumn{2}{|c|}{ Dep. }} & \multirow{2}{*}{ Ind. } & \multicolumn{2}{|c|}{$\beta^{\star}$} & \multicolumn{2}{|c|}{ Regulator } & \multicolumn{2}{|c|}{$\begin{array}{c}\text { Demand } \\
\text { complementarity }\end{array}$} & \multicolumn{2}{|c|}{$\begin{array}{c}\text { Cost } \\
\text { (mmWave) }\end{array}$} & \multicolumn{2}{|c|}{$\begin{array}{c}\text { Cost } \\
\text { (micro LTE) }\end{array}$} & \multicolumn{2}{|c|}{$\begin{array}{l}\text { Fungibility } \\
\text { (small cells) }\end{array}$} & \multicolumn{2}{|c|}{$\begin{array}{c}\begin{array}{c}\text { Fungibility } \\
\text { (micro LTE (S)) }\end{array} \\
\end{array}$} & \multirow[t]{2}{*}{$\mathrm{R}^{2}$} \\
\hline & & & coef. & p-val. & coef. & p-val. & coef. & p-val. & coef. & $\mathrm{p}$-val. & coef. & $\mathrm{p}$-val. & coef. & p-val. & coef. & p-val. & \\
\hline \multirow{12}{*}{ 与 } & \multirow{4}{*}{ 容 } & \# bs & .51 & $\checkmark$ & -.51 & $\checkmark$ & -.25 & $\checkmark$ & .09 & $\checkmark$ & .14 & $\checkmark$ & -.01 & $x$ & -.01 & $x$ & .90 \\
\hline & & capacity & .46 & $\checkmark$ & -.48 & $\checkmark$ & -.23 & $\checkmark$ & .13 & $\checkmark$ & .13 & $\checkmark$ & .31 & $\checkmark$ & -.01 & $x$ & .87 \\
\hline & & traffic & .48 & $\checkmark$ & -.48 & $\checkmark$ & -.33 & $\checkmark$ & .09 & $\checkmark$ & .18 & $\checkmark$ & -.02 & $x$ & -.05 & $\checkmark$ & .87 \\
\hline & & clustering & .54 & $\checkmark$ & -.38 & $\checkmark$ & -.15 & $\checkmark$ & .04 & $\checkmark$ & .25 & $\checkmark$ & -.00 & $x$ & -.02 & $x$ & .83 \\
\hline & \multirow{8}{*}{ 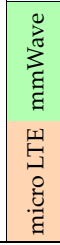 } & \# bs & .23 & $\checkmark$ & -.01 & $x$ & -.14 & $\checkmark$ & -.71 & $\checkmark$ & .06 & $\checkmark$ & .04 & $\checkmark$ & -.00 & $x$ & .75 \\
\hline & & capacity & .22 & $\checkmark$ & -.03 & $x$ & -.12 & $\checkmark$ & -.67 & $\checkmark$ & .05 & $\checkmark$ & .16 & $\checkmark$ & -.15 & $\checkmark$ & .71 \\
\hline & & traffic & .21 & $\checkmark$ & -.01 & $x$ & -.15 & $\checkmark$ & -.72 & $s$ & .07 & $\checkmark$ & .02 & $x$ & -.01 & $x$ & .76 \\
\hline & & clustering & .13 & $s$ & -.02 & $x$ & .04 & $x$ & .31 & $s$ & .07 & $\checkmark$ & .14 & $x$ & -.02 & $x$ & .20 \\
\hline & & \# bs & -.23 & $\checkmark$ & .56 & $\checkmark$ & .11 & $\checkmark$ & -.01 & $x$ & -.34 & $\checkmark$ & .01 & $x$ & -.02 & $x$ & .69 \\
\hline & & capacity & -.23 & $\checkmark$ & .55 & $\checkmark$ & .11 & $\checkmark$ & -.02 & $x$ & -.33 & $\checkmark$ & .01 & $x$ & .11 & $\checkmark$ & .70 \\
\hline & & traffic & -.23 & $\checkmark$ & .62 & $\checkmark$ & .08 & $\checkmark$ & -.01 & $x$ & -.29 & $\checkmark$ & .01 & $x$ & .03 & $x$ & .81 \\
\hline & & clustering & -.25 & $\checkmark$ & .37 & $\checkmark$ & .01 & $x$ & -.01 & $x$ & -.32 & $\checkmark$ & .00 & $x$ & -.10 & $\checkmark$ & .51 \\
\hline \multirow{12}{*}{$\stackrel{0}{z}$} & \multirow{4}{*}{3} & \# bs & -.05 & $\sqrt{ }$ & -.78 & $\checkmark$ & .02 & $\sqrt{ }$ & .10 & $\checkmark$ & .62 & $\checkmark$ & .00 & $x$ & -.05 & $\sqrt{ }$ & .97 \\
\hline & & capacity & -.06 & $\checkmark$ & -.72 & $\checkmark$ & .02 & $\checkmark$ & .12 & $\checkmark$ & .57 & $\checkmark$ & .34 & $\checkmark$ & -.06 & $\checkmark$ & .94 \\
\hline & & traffic & .08 & $\checkmark$ & -.64 & $\checkmark$ & .05 & 5 & .09 & $\checkmark$ & .73 & $\checkmark$ & .02 & $\checkmark$ & -.07 & 5 & .96 \\
\hline & & clustering & .57 & $\checkmark$ & -.26 & $\checkmark$ & -.15 & $\checkmark$ & .05 & $\checkmark$ & .30 & 5 & -.00 & $x$ & -.02 & $x$ & .78 \\
\hline & \multirow{4}{*}{$\begin{array}{l}\text { ट्र } \\
\text { हू } \\
\text { हू }\end{array}$} & \# bs & .01 & $x$ & -.21 & $\checkmark$ & .01 & $x$ & -.72 & $\checkmark$ & .20 & $\checkmark$ & -.00 & $x$ & -.01 & $x$ & .80 \\
\hline & & capacity & .02 & $x$ & -.20 & $\checkmark$ & .00 & $x$ & -.71 & $\checkmark$ & .19 & $\checkmark$ & .11 & $\checkmark$ & -.01 & $x$ & .78 \\
\hline & & traffic & .04 & $x$ & -.13 & $\checkmark$ & -.00 & $x$ & -.74 & $\checkmark$ & .20 & $\checkmark$ & .00 & $x$ & -.02 & $x$ & .82 \\
\hline & & clustering & -.14 & $\checkmark$ & -.38 & $\checkmark$ & .03 & $x$ & -.43 & $\checkmark$ & .29 & $\checkmark$ & .01 & $x$ & .00 & $x$ & .44 \\
\hline & \multirow{4}{*}{ 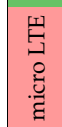 } & \# bs & -.07 & $\checkmark$ & .87 & $\checkmark$ & .01 & $\checkmark$ & -.00 & $x$ & -.37 & $\checkmark$ & -.00 & $x$ & -.02 & $\checkmark$ & .98 \\
\hline & & capacity & .05 & $\checkmark$ & .86 & $\checkmark$ & -.00 & $x$ & -.00 & $x$ & -.47 & $\checkmark$ & -.06 & $\checkmark$ & .10 & $\checkmark$ & .96 \\
\hline & & traffic & -.03 & $\checkmark$ & .89 & $\checkmark$ & .01 & $\checkmark$ & .00 & $x$ & -.40 & $\checkmark$ & -.02 & $s$ & .04 & $\checkmark$ & .98 \\
\hline & & clustering & .13 & $\checkmark$ & .71 & $\checkmark$ & -.01 & $x$ & .01 & $x$ & -.61 & $s$ & -.02 & $x$ & -.01 & $x$ & .73 \\
\hline
\end{tabular}

It represents the mean change in the dependent variable for 1 unit of change in the corresponding independent variable while holding the others.

${ }_{* * * *}^{*}$ indicates statistical significance $(\mathrm{p}$-value $<.01)$ while $\boldsymbol{X}$ indicates not statistically significant ( $\mathrm{p}$-value $\left.>.01\right)$.

${ }_{* * * * *}^{*}$ We omit the standard error and the $95 \%$ confidence interval in order to keep the table more readable.

${ }^{* * * * *}$ The colors are consistent with Fig. 8, Fig. 9, and Fig. 10 in order to help the reader to follow the discussion.

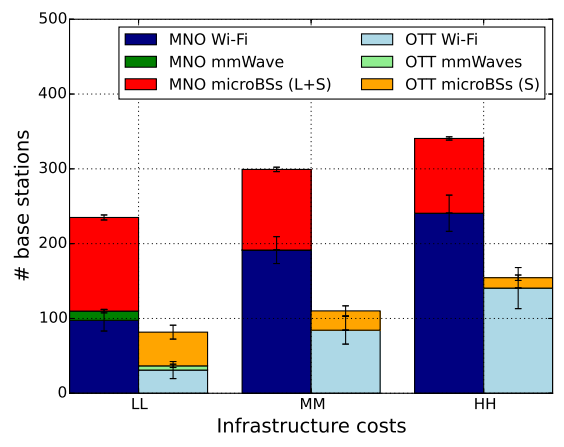

(a)

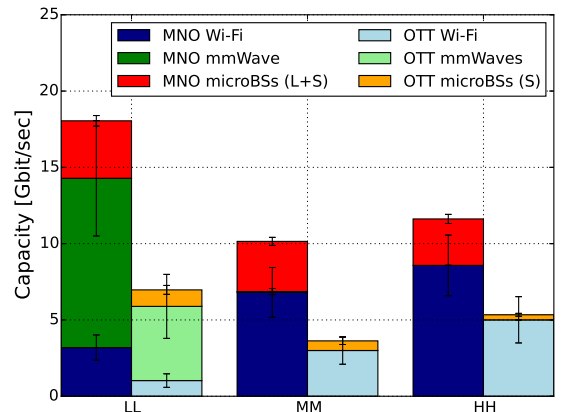

Infrastructure costs

(b)

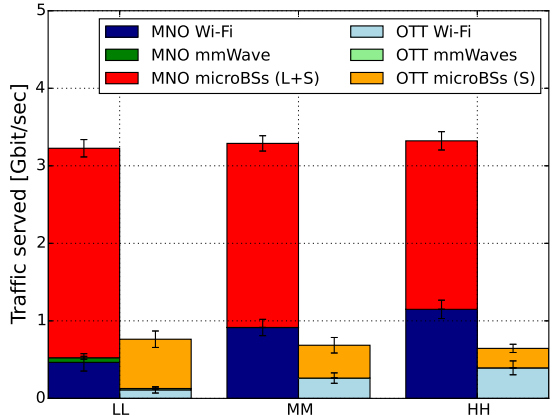

Infrastructure costs

(c)

Fig. 8: Number of base stations deployed (a), capacity supplied (b), traffic served (c) for different combinations of prices. The error bars indicate the $95 \%$ confidence interval.

demand of content $\hat{c}$. Instead the capacity deployed in the reference scenario complements the one deployed by the MNO rather than overlapping with it.

When we lower the complementarity to its minimum value, in Fig. 12, an entirely different picture emerges. The demand for content $\hat{c}$ (Fig. 12(a)) is distributed over a wider area, mostly in the South. As we can see from Fig. 12(b), the OTT deploys a much lower number of base stations, in locations throughout the topology. Also notice from Fig. 12(c) how some of the demand for content $\hat{c}$ is also served by the MNO.

In summary, Fig. 11 and Fig. 12, obtained for different values of complementarity, show two very different networks. In Fig. 11, OTT and MNO complement each other in serving the traffic demand; in Fig. 12 the MNO builds a high-capacity network with vast coverage and the OTT pays a (moderate) fee to use it.

\section{Related WORK}

A first body of works our paper relates to deals with the planning of mobile networks. Particular emphasis is put on their different conditions and requirements with respect to traditional LTE networks, including radically different outdoor and indoor scenarios [36], ultra-dense deployments [37], and device-to-device communications [38].

Much attention has been devoted to new physical-layer technology that, on the one hand, will enable future networks to serve their increased data demand and, on the other hand, will call for new architectures and management strategies. Prominent examples include massive MIMO and millimeter waves [39]. Especially interesting from our viewpoint are the studies that try to quantify the capacity offered by such base stations, including [19], [20], and their cost, e.g., [17], [18]. Cost information is generally scarce, and more easily found in business reports [17] than in peer- 


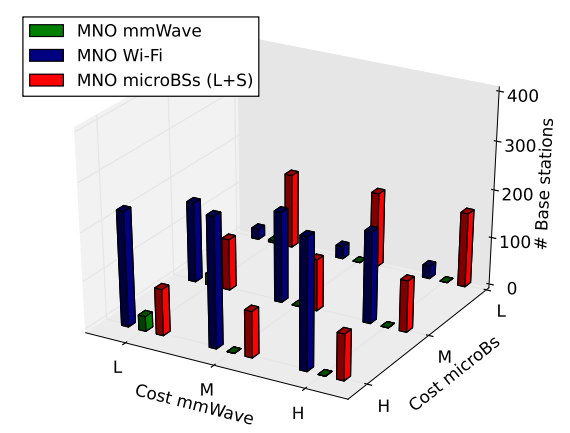

(a)

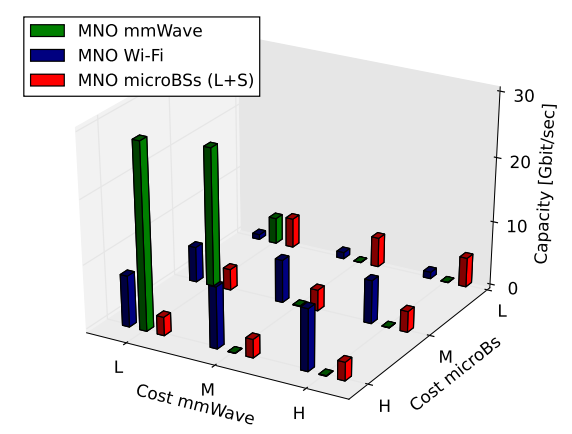

(b)

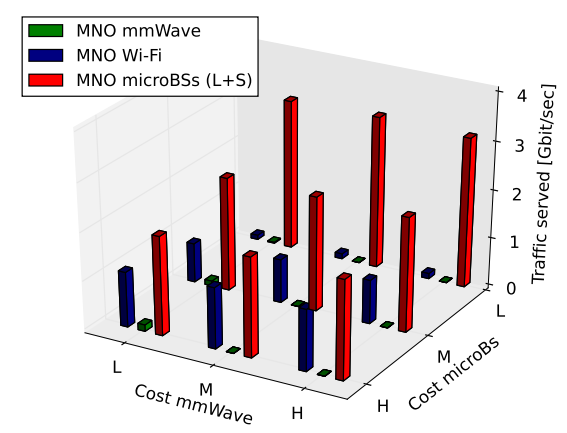

(c)

Fig. 9: MNO. Number of base stations deployed (a), capacity supplied (b), traffic served (c) for all costs combinations.

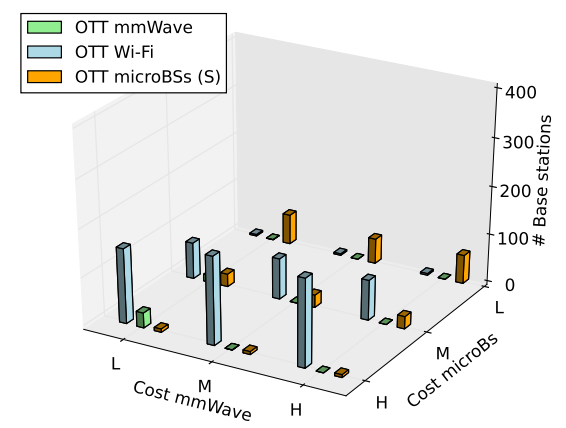

(a)

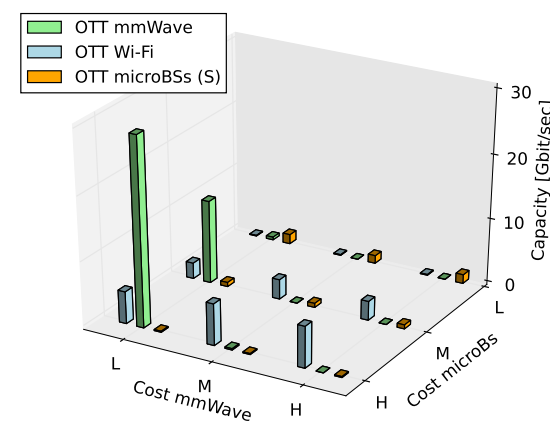

(b)

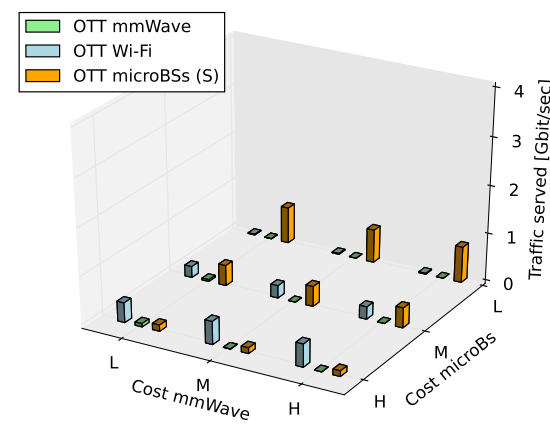

(c)

Fig. 10: OTT. Number of base stations deployed (a), capacity supplied (b), traffic served (c) for all costs combinations.

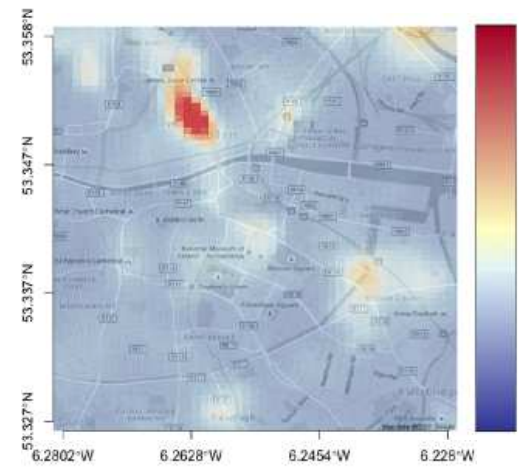

(a)

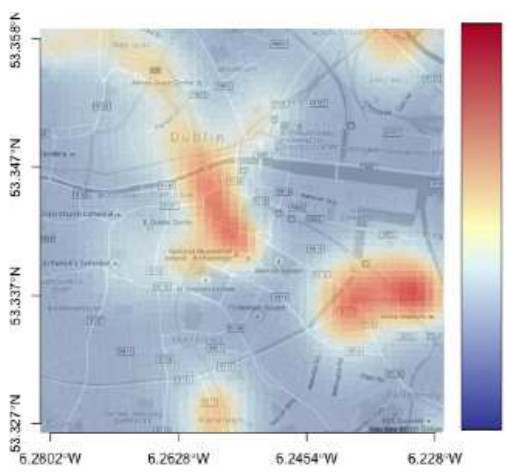

(b)

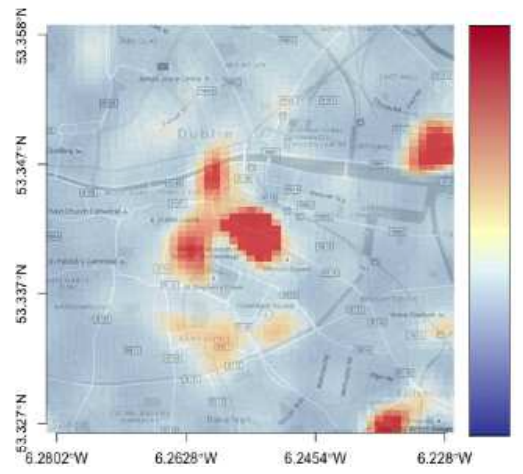

(c)

Fig. 11: High complementarity: location of the demand for content type $\hat{c}(a)$; capacity deployed by the OTT (b) and the MNO (c).

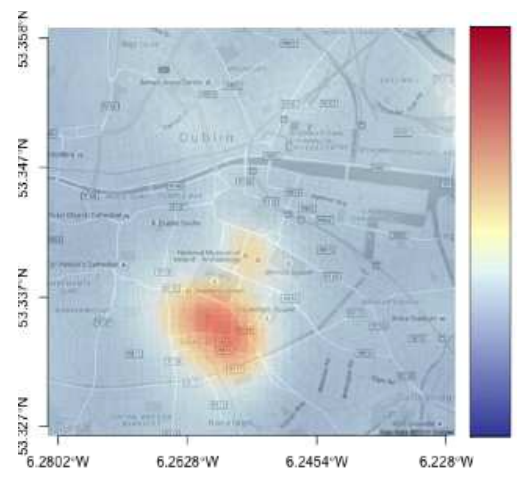

(a)

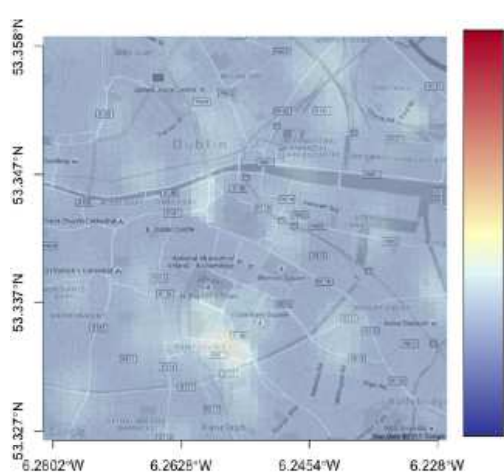

(b)

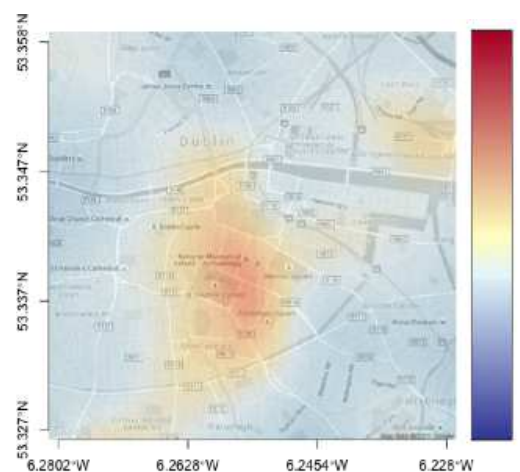

(c)

Fig. 12: Low complementarity: location of the demand for content type $\hat{c}$ (a); capacity deployed by the OTT (b) and the MNO (c). 
reviewed literature.

Fungibility studies are also very relevant to our work. Examples include [8], which introduced several methods to compute fungibility scores, and [40], where those scores are refined. A set of related works aims at the more general objective of assigning a value to wireless spectrum, for the purpose of introducing fluid networks [41], and enabling spectrum trading [42], [43]. The ultimate purpose is evaluating the effectiveness of dynamic spectrum use, where [44] specifically accounts for regulatory decisions.

Finally, our work is related to such real-world measurements of data demand as [34], [45], [46]. Two of these reports show that different types of data demand tend to be spatially correlated [45], [46]. These studies were concerned with web services and smartphone apps only; however, similar conclusions were drawn when more general mobile demand was considered, as in [34], where the authors operated on flow-level information sent to and from cellular devices. Their study reveals that various data applications are not equally popular across all cells, and that "the popularity of some applications is more skewed than others across cells". Moreover, a few applications dominate others given their relative traffic volume, and applications can be grouped into traffic profiles that describe application usage distribution for any given cell. Other works, e.g., [47], [48] have studied real-world deployments of cellular networks, and found that some spacial features thereof are remarkably similar throughout mobile operators and cities.

\section{CONCLUSION}

This paper sought to study the mobile network expansion driven by the OTT's service needs and to assess, first, the factors that are likely to impact deployment decisions by OTTs and MNOs, and second, the characteristics of the resulting networks.

We found that the factors with the deepest influence on the final network are not technical but rather economic (e.g., the cost of base stations) and regulatory (e.g., the type of spectrum and technologies OTTs are allowed to use). Different combinations of these factors yield radically different networks: in some cases, OTTs and MNOs deploy separate networks that complement each other; in others, MNOs find it convenient to offer plentiful, cheap capacity to OTTs, which have no incentive to deploy their own networks. Furthermore, the technologies providing most of the network capacity (most notably, mmWave) will not serve most of the traffic, leaving plenty of opportunities for innovative applications such as proximity services and M2M.

Real-world datasets such as ours are difficult to come by, and one might wonder how general our conclusions based on Dublin city are. While a definite answer would require more datasets, the authors of [48] found that base station deployments of different operators in different cities have remarkably similar spatial properties and can be modeled with very similar statistic models. This, along with the fact that we study different demand profiles with different complementarity values, affords to our study a high level of generality. Indeed, our conclusions about the importance of MNO fees are consistent with the findings of [49], which uses stochastic distributions for both demand and infrastructure.

\section{REFERENCES}

[1] R. Xia et al., "Business Models in the Mobile Ecosystem," in Conference on Mobile Business and Global Mobility Roundtable, 2010.

[2] Value Partners, "Mobile 2015: New Spectrum, Different Business Models, More Competition?" [Online]. Available: http://www.valuepartners.com/downloads/PDF_ Comunicati/value-partners-120210-mobile-2015-henry-alty.pdf

[3] T. Giles et al., "Cost Drivers and Deployment Scenarios for Future Broadband Wireless Networks - Key Research Problems and Directions for Research," in IEEE Vehicular Technology Conference Spring, 2004.

[4] Project Fi - Google. [Online]. Available: https://fi.google.com/

[5] Facebook Inc. [Online]. Available: https://info.internet.org/en/ story/free-basics-from-internet-org/

[6] S. Datoo, "Twitter's latest deal points to ambitions in emerging markets," The Guardian, 2013.

[7] H. Zhang et al., "Coexistence of Wi-Fi and Heterogeneous Small Cell Networks Sharing Unlicensed Spectrum," IEEE Communications Magazine, 2014.

[8] M. Weiss et al., "When is Electromagnetic Spectrum Fungible?" in IEEE New Frontiers in Dynamic Spectrum Access Networks, 2012.

[9] M. Nawrocki et al., Understanding UMTS Radio Network Modelling, Planning and Automated Optimisation: Theory and Practice. John Wiley \& Sons, 2006.

[10] Credit Suisse, "U.S. Wireless Networks Running at $80 \%$ of Capacity," 2011. [Online]. Available: http://benton.org/node/ 81874

[11] Y. J. Chiang et al., "Performance and Cost-Effectiveness Analyses for Cloud Services Based on Rejected and Impatient Users," IEEE Transactions on Services Computing, vol. 9, no. 3, pp. 446-455, 2016.

[12] A. Caprara et al., "Algorithms for the set Covering Problem," Annals of Operations Research, vol. 98, no. 1-4, pp. 353-371, 2000.

[13] F. C. Gomes et al., "Experimental Analysis of Approximation Algorithms for the Vertex Cover and Set Covering Problems," Computers $\mathcal{E}$ Operations Research, vol. 33, no. 12, pp. 3520-3534, 2006.

[14] R. P. Brent, Algorithms for minimization without derivatives. Englewood Cliffs, N.J: Prentice-Hall, 1972.

[15] Google Inc. [Online]. Available: http://www.google.com/loon/

[16] "LTE Technical Modelling Revised Methodology," OFCOM White Paper, 2012. [Online]. Available: http://stakeholders.ofcom.org.uk/binaries/consultations / award-800mhz/annexes/annex14.pdf

[17] M. Paolini, "The Economics of Small Cells and Wi-Fi offload," Senza Fili Consulting, Tech. Rep., 2012.

[18] E. Hossain, "Evolution Toward 5G Cellular Networks: A Radio Resource and Interference Managment Perspective," in IEEE International Conference on Communications (ICC) Tutorials, 2014.

[19] S. Rangan et al., "Millimiter Wave Cellular Wireless Networks: Potentials and Challenges," Proceedings of the IEEE, 2014.

[20] T. S. Rappaport et al., "Millimeter-Wave Urban Channels Communications: Channel Models, Capacity Limits, Challenges and Opportunities," IEEE Communications Theory Workshop, 2014.

[21] E. Perahia and R. Stacey, Next generation wireless LANs : $802.11 n$ and 802.11ac. Cambridge University Press, 2013.

[22] 3GPP Technical Report 36.814, "Further Advancements for EUTRA Physical Layer Aspects," 2010.

[23] ITU. Recommendation ITU-R P.1411-1. https://www.itu.int/ dms_pubrec/itu-r/rec/p/R-REC-P.1411-1-200102-S!!PDF-E.pdf.

[24] K. Lee et al., "Mobile Data Offloading: How Much Can Wi-Fi Deliver?" Networking, IEEE/ACM Transactions on, vol. 21, no. 2, pp. 536-550, 2013.

[25] I. Macaluso et al., "Complexity of Spectrum Activity and Benefits of Reinforcement Learning for Dynamic Channel Selection," Selected Areas in Communications, IEEE Journal on, vol. 31, no. 11, pp. 2237-2248, 2013.

[26] J. Illian et al., Statistical Analysis and Modelling of Spatial Point Patterns (Statistics in Practice), 1st ed. Wiley-Interscience, 2008.

[27] N. Alok et al., "AdTorrent: Digital Billboards for Vehicular Networks," in IEEE/ACM Workshop on Vehicleto-Vehicle Communications, 2005. 
[28] C. Borgiattino et al., "Advertisement delivery and display in vehicular networks," in Vehicular Technology Conference (VTC Fall), 2015 IEEE 82nd, Sept 2015, pp. 1-5.

[29] F. Malandrino et al., "The Price of Fog: a Data-Driven Study on Caching Architectures in Vehicular Networks," in ACM MobiHoc Workshop on the Internet of Vehicles and Vehicles on Internet, 2016.

[30] CNN, "Pokemon Go breaks Apple download records." [Online]. Available: http://money.cnn.com/2016/07/22/technology/ pokemon-go-apple-download-records /

[31] "Irish CSO census data." [Online]. Available: http://www.cso.ie/ en/census/census2011_boundaryfiles/

[32] [Online]. Available: http://www.askcomreg.ie/mobile/ siteviewer.273.LE.asp

[33] Cisco, "Cisco Visual Networking Index: Global Mobile Data Traffic Forecast Update, 2013-2018." [Online]. Available: http:// www.cisco.com/c/en/us/solutions/collateral/service-provider/ visual-networking-index-vni/white_paper_c11-520862.pdf

[34] M. Shafiq et al., "Geospatial and Temporal Dynamics of Application Usage in Cellular Data Networks," IEEE Transactions on Mobile Computing, 2014.

[35] N. R. Draper and H. Smith, Applied Regression Analysis. WileyInterscience, 1998.

[36] C.-X. Wang et al., "Cellular architecture and key technologies for $5 \mathrm{~g}$ wireless communication networks," Communications Magazine, IEEE, vol. 52, no. 2, pp. 122-130, February 2014.

[37] P. Agyapong et al., "Design considerations for a 5G network architecture," IEEE Communications Magazine, vol. 52, no. 11, pp. 65-75, 2014.

[38] F. Malandrino et al., "Toward D2D-Enhanced Heterogeneous Networks," IEEE Communications Magazine, vol. 52, no. 11, pp. 94-100, 2014.

[39] S. Sun et al., "Mimo for Millimeter-Wave Wireless Communications: Beamforming, Spatial Multiplexing, or Both?" IEEE Communications Magazine, vol. 52, no. 12, pp. 110-121, 2014.

[40] M. Gomez and M. Weiss, "How do Limitations in Spectrum Fungibility Impact Spectrum Trading?" in Telecommunications Policy Research Conference, 2013.

[41] L. Doyle and T. Forde, "Towards a Fluid Spectrum Market for Exclusive Usage Rights," in IEEE New Frontiers in Dynamic Spectrum Access Networks, 2007.

[42] C. E. Caicedo and M. B. H. Weiss, "The Viability of Spectrum Trading Markets," in IEEE New Frontiers in Dynamic Spectrum Access Networks, 2010.

[43] L. McKnight et al., "Best Effort versus Spectrum Markets: Wideband and Wi-Fi versus 3G MVNOs?" 2001. [Online]. Available: http://hdl.handle.net/1721.1/1491

[44] J. Zander et al., "On the Scalability of Cognitive Radio: Assessing the Commercial Viability of Secondary Spectrum Access," IEEE Wireless Communications, vol. 20, no. 2, pp. 28-36, 2013.

[45] I. Trestian et al., "Measuring Serendipity: Connecting People, Locations and Interests in a Mobile 3G Network," in ACM/SIGCOMM Conference on Internet measurement, 2009.

[46] Q. Xu et al., "Identifying Diverse Usage Behaviors of Smartphone Apps," in ACM/SIGCOMM Conference on Internet measurement, 2011.

[47] J. Kibiłda and L. A. DaSilva, "Efficient Coverage through Interoperator Infrastructure Sharing in Mobile Networks," in Wireless Days, 2013.

[48] J. Kibiłda et al., "Modelling Multi-operator Base Station Deployment Patterns in Cellular Networks," IEEE Transactions on Mobile Computing, vol. 15, no. 12, pp. 3087-3099, 2016.

[49] J. Kibiłda et al., "Incentives for Infrastructure Deployment by Over-the-Top Service Providers in a Mobile Network: A Cooperative Game Theory Model," IEEE International Conference on Communications, 2016.

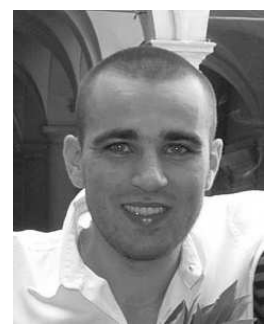

Paolo Di Francesco Paolo Di Francesco is currently a Software Developer at RadarServices Smart-IT Security GmbH. Previously he was a post-doc at CTVR/CONNECT Trinity College Dublin, Ireland, where he obtained his $\mathrm{PhD}$ in 2015 . He received the B.S., M.S. degree in telecommunications engineering from University of Bologna in 2008 and 2011 respectively. His research interests include resource sharing, network optimization, Software Defined Radios, data-analysis, and network security.

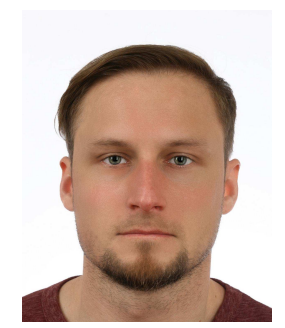

Jacek Kibiłda Jacek Kibiłda received the M.Sc degree from Poznań University of Technology, Poland, in 2008, and the Ph.D. degree from Trinity College, The University of Dublin, Ireland, in 2016. From 2009 to 2010 he worked at Nokia Siemens Networks and from 2010 to 2012 at EIT+ ICT Research Center, both in Wrocław, Poland. Currently he is a research fellow with CONNECT, Trinity College, The University of Dublin, Ireland. His research focuses on architectures and models for future mobile networks.

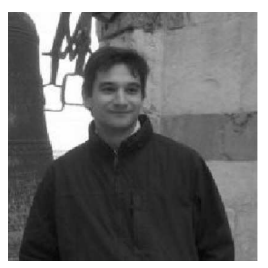

Francesco Malandrino Francesco Malandrino earned his Ph.D. in 2012 from Politecnico di Torino, Italy, where he is currently a post-doc. Before his current appointment, he held shortterm positions at Trinity College, Dublin, and at the Hebrew University of Jerusalem as a Fibonacci Fellow. His interests focus on wireless and vehicular networks and infrastructure management.

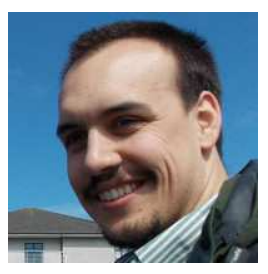

Nicholas Kaminski Nicholas J. Kaminski conducts research focused on extending the bounds of wireless technology by deploying targeted intelligence to act in harmony with flexible radio systems. He advances distributed radio intelligence through experimentation-based research and furthers wireless communications by incorporating techniques for understanding communications from complex systems science. Dr. Kaminski received his masters degree in Electrical Engineering in 2012 and his Ph.D. in 2014 from Virginia Tech, USA. During this time he was funded as a Bradley Fellow at the Bradley Department of Electrical and Computer Engineering at Virginia Tech, USA

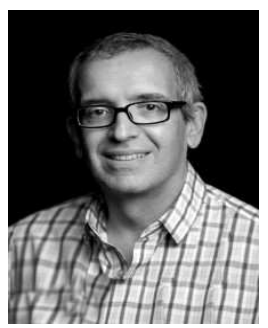

Luiz A. DaSilva Luiz A. DaSilva holds the chair of Telecommunications at Trinity College Dublin. He also has an adjunct research professor appointment in the Bradley Department of Electrical and Computer Engineering, Virginia Tech. His research focuses on distributed and adaptive resource management in wireless networks, and in particular wireless resource sharing, dynamic spectrum access, and the application of game theory to wireless networks. He is currently a principal investigator on research projects funded by the National Science Foundation in the United States, the Science Foundation Ireland, and the European Commission under Horizon 2020 and Framework Programme 7. He is a co-principal Investigator of CONNECT, the Telecommunications Research Centre in Ireland. He is a fellow of the IEEE, for contributions to cognitive networking and resource management for wireless networks, and an IEEE Communications Society distinguished lecturer. 